\section{CT-07 A FAITH-BASED APPROACH TO INCREASING AFRICAN AMERICAN AWARENESS AND PARTICIPATION IN LUPUS CLINICAL TRIALS}

Patricia B Davidson*, R Paola Daly. Lupus Foundation of America Inc., USA

\subsection{6/lupus-2018-Ism.79}

Background Improving Minority Participation and Awareness in Clinical Trials (IMPACT) for Lupus was a 2016-2017 initiative through the Lupus Foundation of America aimed at increasing participation of African Americans in lupus clinical trials. A faith-based educational model using health navigators for reaching this population through churches was created by leveraging existing community partnerships and by employing culturally relevant communication tools and outreach strategies. IMPACT for Lupus used a community-based participatory research (CBPR) approach and incorporated Ford's conceptual framework ${ }^{3}$ focusing on awareness as the fundamental first step to clinical trial participation.

Methods First, an Action Partnership (AP) of public and private stakeholders, including community leaders and a patient representative, was created to provide input throughout the entire project. Next, an extensive literature review of past studies and initiatives to increase clinical trials awareness served as formative research to develop a mixed-methods study assessing the receptivity and cultural appropriateness of the educational model (see figure 1). Semi-structured interviews were conducted among 20 key informants. A survey was developed for patients $(n=205)$ and physicians $(n=122)$. Patient materials were assessed for cultural relevance through a community advisory board (CAB). A three-month pilot testing of the model was implemented and evaluated in three sites: Charleston, SC; Atlanta, GA; and Fort Washington, MD. Five outcome measures were developed to evaluate receptivity to the model through pre- and post-patient surveys and navigator interviews.

Results There was unanimous agreement among physicians, community leaders, and patients to utilize a collaborative approach including physicians, nurses, community based organizations, or other intermediaries, to enhance patient consideration of clinical trials. Physicians and community leaders recommended a community-based navigator to facilitate the relationship and indicated that the most effective approach to outreach to the African American community is through the church. Among patients, more than half also ranked the church as a preferred place to learn about lupus clinical trials. 95\% of physicians indicated that lupus patients do not inquire about clinical trials very often or at all. However, patient communication with their healthcare providers was significantly associated with clinical trial participation. Pilot evaluation showed that navigator outreach through churches resulted in increased patient-provider communication about clinical trials.

Conclusions IMPACT for Lupus showed promise as an effective faith-based education model to raise awareness of clinical trials among African Americans with lupus. Additional studies with extended implementation periods and a focus on smaller, local churches are needed to support the findings of this study.

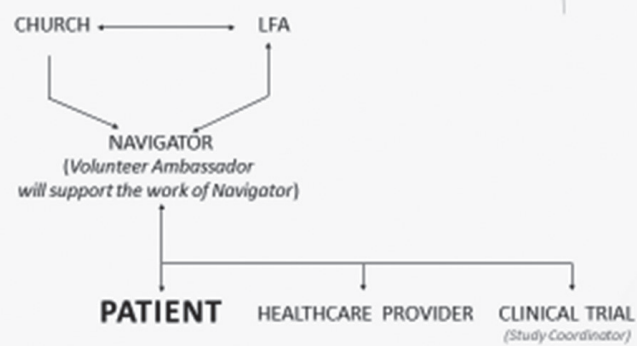

Abstract CT-07 Figure 1 IMPACT for lupus model

Acknowledgements IMPACT for Lupus Action Partnership (AP); Tufts Center for the Study of Drug Development (CSDD); Medical University of South Carolina (MUSC); Georgia Chapter of the Lupus Foundation of America; Carlden Inc.

This program was funded by the Office of Minority Health (OMH) grant number CPIMP161122-01-00.

\section{CT-08 SAFETY AND TOLERABILITY OF OMALIZUMAB, A HUMANIZED ANTI-IGE MONOCLONAL ANTIBODY IN SYSTEMIC LUPUS ERYTHEMATOSUS (STOP LUPUS)}

${ }^{1}$ Sarthak Gupta*, 'Sarfaraz Hasni, ${ }^{1}$ Michael Davis, 'Elaine Poncio, 'Y Yenealem TemesgenOyelakin, 1,2Elizabeth Joyal, 'Alice Fike, 'Zerai Manna, ${ }^{3}$ Sungyoung Auh, 'Y Yinghui Shi, ${ }^{1}$ Diana Chan, 'Philip Carlucci, ${ }^{4}$ Ann Biehl, 'Barbara Dema, ${ }^{1}$ Nico Charles, ${ }^{3}$ James Balow, ${ }^{3}$ Meryl Waldman, ${ }^{1}$ Richard Siegel, ${ }^{1}$ Mariana J Kaplan, ${ }^{5}$ Gabor Illei, ${ }^{1}$ Juan Rivera. ${ }^{1}$ National Institute of Arthritis and Musculoskeletal and Skin Diseases, National Institutes of Health, Bethesda, MD, USA; ${ }^{2}$ Present Address, National Institute of Child Health and Human Development, National Institutes of Health, Bethesda, MD, USA; ${ }^{3}$ National Institute of Diabetes and Digestive and Kidney Diseases, National Institutes of Health, Bethesda, MD, USA; ${ }^{4}$ Clinical Center, National Institutes of Health, Bethesda, MD, USA; ${ }^{5}$ National Institute of Dental and Craniofacial Research, National Institutes of Health, Bethesda, MD, USA

\subsection{6/lupus-2018-Ism.80}

Background Previous evidence indicates a putative pathogenic role for IgE autoantibodies in systemic lupus erythematosus (SLE). We hypothesized that Omalizumab, a monoclonal antibody that binds IgE, will reduce SLE activity by decreasing circulating IgE autoantibodies, subsequently blocking basophil activation and type I IFN responses. This study assessed the safety, tolerability, biologic and clinical efficacy of Omalizumab in mild to moderate SLE.

Methods Fifteen subjects with SLE and a Systemic Lupus Erythematosus Disease Activity Index 2000 (SLEDAI 2K) of $\geq 4$ and elevated $\mathrm{IgE}$ autoantibodies (above 2SD of mean of healthy controls) were randomized to receive Omalizumab or placebo $(2: 1)$ added to their baseline standard of care therapy (excluding monoclonal antibodies and alkylating agents) for 16 weeks. This was followed by a 16 week open label phase and 4 week washout period. SLEDAI 2K, British Isles Lupus Assessment Group index (BILAG 2004) and Physician Global Assessment (PGA) were recorded at each visit. The Systemic Lupus Erythematosus Responder Index (SRI 4) was calculated as a composite measure of improvement in disease activity at 
weeks 16, 32 and 36. Type I Interferon (IFN) induced gene signature was determined using a previously validated four gene interferon score (IFI27, IFI44, IFI44L, RSAD2) using quantitative PCR.

Results No local or systemic allergic reactions occurred following Omalizumab injections. Of the 52 adverse events (AEs), 49 were reported as mild while 3 met the criteria for severe AEs. SLEDAI $2 \mathrm{~K}$ scores improved significantly in the Omalizumab group at week $16(p=0.038)$, as well as during the open label phase in subjects initially receiving placebo $(p=0.020)$. No worsening in BILAG scores or PGA were detected. A total of $26 \%(4 / 15)$ patients achieved SRI4 response criteria with Omalizumab. IFN gene signature improved in subjects treated with Omalizumab $(p=0.11)$, especially in subjects with high baseline IFN scores $(\mathrm{p}=0.052)$.

Conclusions Omalizumab in subjects with SLE is well tolerated and associated with improvement in disease activity potentially linked, at least in part, to modulation of type-I IFN responses. Larger randomized clinical trials are required to assess the role of this drug in patients with SLE.

Trial registration

Clinical Trials registration number NCT01716312

\section{Environmental Factors}

\section{EF-01 SOCIOECONOMIC DETERMINANTS AND SLE SEVERITY IN BLACK PATIENTS IN BARBADOS}

Cindy Flower*. University of the West Indies, Cave Hill campus, Barbados

\subsection{6/lupus-2018-Ism.81}

Background SLE patients of lower socioeconomic position (SEP) exhibit increased morbidity and mortality but less clear is whether the disease incidence is increased in the setting of poverty and whether a more severe disease phenotype develops in poorer patients. Crucial etiologic factors for SLE may predominate in genetically-predisposed individuals of lower SEP. Lack of running water at age 12 and frequent childhood infections- both of which are associated with poverty, have been implicated in some groups. In our cohort of black patients in Barbados, we sought to determine by comparison with the general population if SLE clusters in persons of lower SEP and if poorer patients had a more severe disease phenotype.

Methods Patients from the Barbados National Lupus Registry (universal access to care) were included if they had a clinical diagnosis of SLE made by a rheumatologist in the 7 year period 2008-2014.

The clinical status was assessed at the end of 2017 ensuring all patients had at least 3 years of follow up - the period of time within which most severe SLE organ involvement develops. Private health insurance coverage was used a proxy for SEP. Patients were divided into 2 groups based on the presence or absence of health insurance at disease diagnosis. We investigated the effect of SEP on major organ involvement (yes/no), SLE nephritis (yes/no), neuropsychiatric disease (yes/ no), and vital status in December 2017 (alive, alive with chronic kidney disease, died).

Results In a subset of 135 patients with SLE $(F=126), 29$ (21\%) had private healthcare insurance at diagnosis (median age 32), the remaining 106 (79\%) did not (median age 35).
This compares to a national prevalence of private healthcare of $24 \%$ (95\% CI $21 \%$ to $26 \%$ ). The association between health insurance status and each of the four complications/outcomes is presented in table 1 . After adjusting for disease duration, patients without private health insurance at diagnosis had more complicated SLE and worse SLE outcomes (Odds Ratios $>1$ ) in all cases. For two of these clinical complications (major organ involvement and SLE nephritis) the effects were borderline statistically significant at the 5\% level.

Conclusions Socioeconomic determinants appear to affect the severity of SLE in Barbados with poorer patients having a more severe disease phenotype.

The high morbidity and mortality documented in the international literature in the setting of poverty may be due to a combination of social issues and the presence of more aggressive disease from the outset.

\begin{tabular}{|c|c|c|c|}
\hline Outcome & Odds ratio & $95 \% \mathrm{Cl}$ & $P$ value \\
\hline Major organ involvement $\dagger$ & 2.69 & $1.00-7.26$ & 0.05 \\
\hline Lupus nephritis $\dagger$ & 3.24 & $1.01-10.37$ & 0.05 \\
\hline Neuropsychiatric lupus $\dagger$ & 4.40 & $0.55-35.16$ & 0.16 \\
\hline Vital status & 3.83 & $0.84-17.45$ & 0.08 \\
\hline
\end{tabular}

\section{EF-02 LONGITUDINAL STUDY OF LONG-TERM POVERTY AND PERSISTENT DEPRESSIVE SYMPTOMS IN SLE}

${ }^{1}$ Ed Yelin*, ${ }^{1}$ Jinoos Yazdany, ${ }^{1}$ Laura Trupin, ${ }^{2}$ Natalie McCormick, ${ }^{1}$ Patricia Katz. ${ }^{1}$ University of California, San Francisco, San Francisco, USA; ${ }^{2}$ University of British Columbia, Vancouver and Arthritis Research Centre of Canada

\subsection{6/lupus-2018-Ism.82}

Background A prior study found that persons with SLE in longterm poverty have greater accumulation of disease damage over 6 years than those exiting poverty or never in poverty. ${ }^{1}$ The present study evaluates the effect of long-term poverty status on depressive symptoms over the same duration of time.

Methods Data are from the UCSF Lupus Outcomes Study in which persons with SLE were recruited in 2003 throughout the U.S. and interviewed annually through 2015. In each year we characterized respondents' poverty status based on household income and family size and administered the CESD measure of depressive symptoms, defining a high level of depressive symptoms using a validated SLE-specific cutpoint $(\geq 24)$ associated with a formal diagnosis of depression. ${ }^{2}$ Prevalent persistent depression was defined as having high levels of depressive symptoms for $\geq 3$ years between 2009 and 2015. Incident persistent depression used the same criteria, measured only among those who had low levels of depressive symptoms between 2006 and 2009. Logistic regression was used to estimate the impact of being poor in every year from 2003-2009, permanently leaving poverty by 2009, or never being poor on prevalent and incident persistent depression, with and without adjustment for gender, age, marital status, race/ethnicity, education, disease duration, extent of accumulated damage by 2009 using the Brief Index of Lupus Damage, ${ }^{3}$ smoking status, and BMI. 